\title{
ONE-DIMENSIONAL KINETIC MODELS OF GRANULAR FLOWS
}

\author{
Giuseppe Toscani ${ }^{1}$
}

\begin{abstract}
We introduce and discuss a one-dimensional kinetic model of the Boltzmann equation with dissipative collisions and variable coefficient of restitution. Then, the behavior of the Boltzmann equation in the quasi elastic limit is investigated for a wide range of the rate function. By this limit procedure we obtain a class of nonlinear equations classified as nonlinear friction equations. The analysis of the cooling process shows that the nonlinearity on the relative velocity is of paramount importance for the finite time extinction of the solution.
\end{abstract}

Mathematics Subject Classification. 76P05, 82C40.

Received: July 11, 2000. Revised: October 11, 2000.

\section{INTRODUCTION}

Granular materials consist of a large number of small discrete grains. The individual grains interact by nearly instantaneous collisions, much like in the classical model of a gas. Since the grains must be cohesionless, they should only interact like hard-spheres, without long-range forces of any kind.

The crucial difference between collisions of granular and ideal gas particles, relies in the intrinsic inelasticity of the collisions between grains. The lost energy heats the grains, but, unlike a classical fluid, this energy is not returned in the form of random motion, or heat, of the grains themselves. In this sense, there is dissipation of energy. The dissipative nature of the particle collisions will bring a perturbed system quickly to the rest.

For identical particles, the energy lost in a collision is usually expressed by the loss of center of mass velocity as

$$
\mathbf{v}^{\prime}-\mathbf{w}^{\prime}=-h(\mathbf{v}-\mathbf{w})
$$

where $\mathbf{v}, \mathbf{w}$ and $\mathbf{v}^{\prime}, \mathbf{w}^{\prime}$ are the velocities of two grains before and after a collision respectively, and $0 \leq h \leq 1$ is the coefficient of restitution. Perfectly elastic collisions correspond to $h=1$, while perfectly inelastic collisions are obtained if $h=0$. In this latter case the particles come out of the collision with the same velocity.

Granular materials composed by a large number of grains whose size is of a few microns are mesoscopic in nature [12], and the natural way to describe their evolution is to use methods borrowed from the kinetic theory of rarefied gases [8] and appropriately modified. This approach is at the basis of many recent studies (see $[4,10,12,17]$ and the references therein), where the basic models are Boltzmann-like equations for hard-spheres undergoing partially inelastic collisions, with a fixed coefficient of restitution $h$. Like in the classical Boltzmann equation [8], the collision kernel is proportional to the relative velocity $\left|v^{\prime}-w^{\prime}\right|$.

Two phenomena are peculiar to granular materials: collapse and clustering. The first is a kinetic and the second is a hydrodynamic phenomenon. They are results of the inelastic nature of these systems and have no

Keywords and phrases. Granular gases, Boltzmann equation, long-time behavior of solutions.

1 Dipartimento di Matematica, Università di Pavia, 27100 Pavia, Italy. e-mail: toscani@dimat.unipv.it

(c) EDP Sciences, SMAI 2000 
equivalent in atomic gases. The essence of the collapse phenomenon can be easily understood by observing a single inelastic ball dropped onto a surface. It will come to rest in a finite time, but it will have experienced (at least, in principle) an infinite number of bounces before it does so. A similar effect occurs in many-body systems, where the surface is replaced by a collection of particles and where relative velocities of particles become very small, leading (via a theoretically infinite number of collisions) to the emergence of strings of particles whose relative velocities vanish. This mechanism is responsible of noticeably difficulties in numerical simulations since a very large number of collisions occur in finite time while nothing much changes in the system [12].

In all the kinetic models quoted above $[4,10,12,17]$, inelastic collisions are described by a fixed coefficient of restitution $h$. This choice, while absolutely reasonable from the point of view of the mathematical complexity of the model, appears inadequate to describe the whole variety of behaviors of these materials.

More accurate kinetic models could be obtained via a more precise description of the inelastic collisions. Maybe the most natural way to obtain this improvement is to consider collisions between grains in which the coefficient of restitution $h$ can vary. More realistic coefficients of restitution that depend on the relative velocity have been treated in the pertinent literature. For example, a variable restitution coefficient, in such a way that collisions with small relative velocity are close to be elastic, has been recently used in molecular dynamics simulation of oscillated granular media [6]. In another recent paper [11], it has been shown that a variable coefficient of restitution with appropriate behavior for small values of the relative speed prevents inelastic collapses.

In this paper, we propose a (one-dimensional) model of the Boltzmann equation for hard-spheres performing partially inelastic collisions, where the coefficient of restitution $h$ depends on the relative speed. The choice of a one-dimensional Boltzmann equation represents a compromise between the requirement to have a good approximation of dilute granular systems from the physical point of view, and reasonable difficulties of solutions both from the mathematical and numerical point of view [18]. In fact, on the contrary to elastic collisions, partially inelastic collisions have a nontrivial outcome as well in one dimension, and the one-dimensional idealization is a nontrivial adjunct to more realistic studies [16].

By this kinetic model we will try to clarify the role of a variable coefficient of restitution in the collapse (cooling) phenomenon. To do this, we will study two different asymptotic problems related to the spatially homogeneous equation. The first one is a rigorous study of the Boltzmann equation in the quasi elastic limit [17]. As explained in Section 4, in this limit, solutions of the Boltzmann equation are well approximated by solutions of nonlinear friction type equation, in which the nonlinearity is determined by the dependence of the coefficient of restitution on the relative velocity. The second problem is the analysis of the cooling process of the spatially homogeneous granular gas. We study this problem both for the Boltzmann equation and for the nonlinear frictions equations. We find that the type of dependence of the coefficient of restitution on the relative velocity is responsible of completely different behaviors. In accord with the result of [11], it can be shown that no finite inelastic collapse can occur for hard--spheres interactions even if the coefficient of restitution depends on the relative velocity so that grains are close to be inelastic for small relative velocity. On the contrary, the study of the self-similar solutions of the nonlinear friction equations suggests that finite extinction can occur if the coefficient of restitution has an appropriate behavior with respect to the relative velocity.

Some of these problems have been addressed before. In particular, a different kinetic model has been recently proposed in [7]. This model is reminiscent of the Boltzmann equation for Maxwell molecules [8], where the collision kernel does not depend on the relative velocity. A nonlinear friction equation corresponding to hardspheres with constant coefficient of restitution has been studied by $[4,17]$.

\section{The Kinetic MOdeL}

Consider a system of (partially) inelastic point particles, all with the same mass, moving on a line. If we impose that the collisions conserve momentum but dissipate kinetic energy, the post-collisional velocities $v^{\prime}$ and 
$w^{\prime}$ are related to $v$ and $w$ by

$$
v^{\prime}=\frac{1}{2}(v+w)+\frac{1}{2}(v-w) h ; \quad w^{\prime}=\frac{1}{2}(v+w)-\frac{1}{2}(v-w) h .
$$

Here, $h \in[0,1]$ is the coefficient of restitution, and $v^{\prime}-w^{\prime}=h(v-w)$. This notation is consistent with particles that simply cross each other in a perfectly elastic collision, where $h=1$. If $h=0$ the collisions are perfectly inelastic, and the particles come out of the collision with the same velocity. In between these two extremes, the system is a simple model of a one dimensional "granular medium".

As discussed in [12], experimental works show that the coefficient of restitution may depend on the relative velocity. The grains are close to be elastic for binary collisions with a small relative velocity, while they exhibit a certain degree of inelasticity when the relative velocity in the binary collision is high. A simple way to take into account this phenomenon, is to consider the restitution coefficient in the form

$$
h=h(|v-w|, \theta)=\frac{1}{1+\theta|v-w|^{\gamma}},
$$

where the exponent $\gamma$ characterizes the asymptotic of the restitution coefficient with respect to the relative velocity. The variable $\theta \in \mathbb{R}^{+}$furnishes a measure of the degree of inelasticity of the collision, and we will call it by now inelasticity parameter. Purely elastic collisions are obtained for $\theta=0$, while perfectly inelastic collisions correspond to $\theta=+\infty$. For any fixed value of the inelasticity parameter $\theta$, a positive value of $\gamma$ corresponds to grains that are close to be elastic for small relative velocity. Of course, $\gamma<0$ gives the opposite phenomenon, namely the grains are close to be elastic for large relative velocities. We will refer to this case as the case of "anomalous" granular materials. To any collision we associate a kernel $\beta(\theta)|v-w|$ which takes into account both the rate function of the rigid spheres interactions, and the probability that collisions with a degree of inelasticity $\theta$ occur. Let us denote by $v^{*}(v, w)$ and $w^{*}(v, w)$ the pre-collisional velocities corresponding to $v, w$. If $J$ is the Jacobian of the transformation $(v, w) \rightarrow\left(v^{\prime}, w^{\prime}\right)$, a direct computation shows that

$$
J=J\left(\theta|v-w|^{\gamma}\right)=h\left(\theta|v-w|^{\gamma}\right)\left(1-\gamma \theta|v-w|^{\gamma} h\left(\theta|v-w|^{\gamma}\right)\right)
$$

Considering that $\theta|v-w|^{\gamma} h\left(\theta|v-w|^{\gamma}\right) \leq 1$, it turns out that, for all $\gamma<1, J\left(\theta|v-w|^{\gamma}\right)$ is nonnegative. In addition, $J(r)$ is strictly positive on every compact set of $\mathbb{R}^{+}$. Consequently, the pre-collisional velocities $v^{*}$ and $w^{*}$ are well-defined for all $(v, w)$.

With these choices, following the standard procedures of kinetic theory, we introduce the phase-space density function $f(x, v, t)$ defining $f(x, v, t) \mathrm{d} x \mathrm{~d} v$ to be the number of particles located between $x$ and $x+\mathrm{d} x$ with velocities between $v$ and $v+\mathrm{d} v$ at time $t$. If we assume that the probability of multiple collisions is negligible, and that the probability of a binary collision between two particles with velocities $v$ and $w$ involves only the product $f(v) f(w)$, "molecular chaos assumption", the governing equation for $f$ is a one-dimensional Boltzmann equation:

$$
\frac{\partial f}{\partial t}+v \frac{\partial f}{\partial x}=Q_{\gamma}(f, f)(x, v, t)
$$

where $Q_{\gamma}$ is the so-called granular collision operator, which describes the change in the density function due to creation and annihilation of particles in binary collisions:

$$
Q_{\gamma}(f, f)=\int_{\mathbb{R}^{+}} \int_{\mathbb{R}^{+}} \beta(\theta)\left\{\left|v^{*}-w^{*}\right| \frac{1}{J} f\left(v^{*}\right) f\left(w^{*}\right)-|v-w| f(v) f(w)\right\} \mathrm{d} w \mathrm{~d} \theta .
$$

For particular choices both of the function $\beta$ and of the exponent $\gamma$, the collision operator (6) reduces to wellknown models. If $\gamma=0$, and $\beta(\theta)$ equals the Dirac delta function $\delta(\theta-(1-q) / q)$, where $q<1$ is a positive constant, we obtain the Boltzmann equation introduced in $[10,17]$. This equation considers the grains like rigid spheres, and has constant coefficient of restitution $h=q$. 
Remark 2.1. Boltzmann operator (6) has much in common with the one-dimensional collision operator of Kac caricature of a Maxwell gas [13], and its generalization [9]. Kac operator, which is a very popular model in kinetic theory of rarefied gas, reads

$$
Q_{k}(f, f)=\int_{\mathbb{R}} \int_{-\pi}^{\pi} \beta(|\theta|)\left[f\left(v^{\prime}\right) f\left(w^{\prime}\right)-f(v) f(w)\right] \mathrm{d} w \mathrm{~d} \theta
$$

and the post-collisional velocities are given by

$$
v^{\prime}=v \cos \theta-w \sin \theta, \quad w^{\prime}=v \sin \theta+w \cos \theta
$$

while the rate function $\beta(\cdot)$, which is a uniform probability density in the original Kac model [13], is almost general in Desvillette's paper [9]. In particular, in [9] by analogy with the non cut-off kernel of the true Boltzmann equation [8], the function $\beta(\theta)$ has a singularity of the form $\theta^{-\alpha}$ when $\theta \rightarrow 0^{+}$and $1<\alpha<3$.

Mutatis mutandis, both models are based on one-dimensional collisions depending on a given parameter $\theta$, in such a way that mass and momentum are conserved but the energy is dissipated (2), or mass and energy are conserved, but not the momentum (8). The probability of such an outcome is described by a suitable rate function, whose properties have been shown to be significant for the regularity of the solution [9], and for the study of asymptotic problems [19].

Remark 2.2. The choice of the dependence of the coefficient of restitution on the relative velocity we proposed in (3) is largely arbitrary, and could be modified in many ways. Numerical simulation could help to find different and more accurate expressions. Likewise, the properties of the rate function are largely unknown. In view of the analogies, some of these properties can be argued looking at the Kac equation, that has been intensively studied from many years now. In particular, as remarked in [9], if the rate function $\beta(\theta)$ is integrable, the solution to the spatially homogeneous Boltzmann equation (5) retains memory (at any time $T>0$ ) of the initial distribution, while this memory is destroyed (as far as the regularity is concerned) in the non cut-off case. This argument can be used to conclude that, if the rate function $\beta(\theta)$ is integrable, we can not expect collapse in finite time for the solution of the homogeneous Boltzmann equation with dissipative collisions. In consequence of this, in the remaining of this paper we will consider mainly rate functions $\beta(\theta)$ with a non integrable singularity when $\theta \rightarrow 0^{+}(\theta=0$ corresponds to elastic collisions $)$. As for Kac model, this can be done requiring

$$
\beta(\theta) \sim \theta^{-\alpha}
$$

when $\theta \rightarrow 0^{+}$, and $\alpha \in(1,3]$.

Remark 2.3. For grains colliding according to (2) the energy lost in a single collision is

$$
v^{\prime 2}+w^{\prime 2}-\left(v^{2}+w^{2}\right)=-\frac{1}{2}\left(1-h^{2}\right)(v-w)^{2} .
$$

It is clear that any reasonable kernel has to be such that the mean amount of energy transfer is finite. Since

$$
\frac{1}{2}\left(1-h^{2}\right)(v-w)^{2} \leq \frac{\theta}{2}|v-w|^{2+\gamma}
$$

this corresponds to impose the condition

$$
\int_{\mathbb{R}^{+}} \beta(\theta) \theta \mathrm{d} \theta<\infty
$$

As we shall see later on, (12) is the natural condition under which the quasi elastic limit of the Boltzmann equation (5) obtained formally in [17] can be rigorously justified. 


\section{The homogeneous Dissipative Boltzmann Equation}

We will start the theoretical analysis of equation (5) by discussing the main properties of the spatially homogeneous equation,

$$
\frac{\partial f}{\partial t}=Q_{\gamma}(f, f)(v, t)
$$

when the rate function $\beta$ satisfies conditions (9), (12), and $\gamma<1$. Within these conditions, the Jacobian $J$ is well-defined, and the mean amount of energy transfer is finite.

Denote by $\mathcal{M}_{0}$ the space of all probability measures in $\mathbb{R}$ and by

$$
\mathcal{M}_{p}=\left\{\mu \in \mathcal{M}_{0}: \int_{\mathbb{R}}|v|^{p} \mu(\mathrm{d} v)<+\infty, p \geq 0\right\},
$$

the space of all Borel probability measures of finite momentum of order $p$, equipped with the topology of the weak convergence of the measures. Several types of metrics on $\mathcal{M}_{p}$ can be considered (see [25]). For the purposes of this paper we will introduce a class of the so-called minimal metrics.

Let $\mu, \nu$ in $\mathcal{M}_{p}$, and let $\Pi(\mu, \nu)$ be the set of all probability distributions $L$ in $\mathcal{M}_{p}(\mathbb{R} \times \mathbb{R})$ having $\mu$ and $\nu$ as marginal distributions. Let

$$
T_{p}(\mu, \nu)=\inf _{L \in \Pi(\mu, \nu)} \int|v-w|^{p} \mathrm{~d} L(v, w) .
$$

Then $\tau_{p}=T_{p}^{1 / p}$ metrizes the weak-* topology $T W_{*}$ on $\mathcal{M}_{p}$. We note that $T_{1}$ is the Kantorovich-Vasershtein distance of $\mu$ and $\nu[14,21]$. For a detailed discussion, and application of these distances to statistics and information theory, see [20]. The case of particular interest here is $p=2$. This is due to the fact that the a Maxwellian distribution can not be a solution of the inelastic Boltzmann equation (13), while the Dirac delta distribution is obviously in the kernel of the collision operator (6). Let $\mu$ be a probability measure with momentum $M$. If we denote with $\delta(v-M)$ the Dirac measure centered on $M$, it is immediate to conclude that

$$
T_{2}(\mu, \delta)=\inf _{L \in \Pi(\mu, \delta)} \int|v-w|^{2} \mathrm{~d} L(v, w)=\int_{\mathbb{R}}(v-M)^{2} \mu(\mathrm{d} v, t) .
$$

Hence the study of the time-decay of $\int(v-M)^{2} \mu(\mathrm{d} v, t)$ gives at the same time the decay in the $\tau_{2}$-metric of the solution to (13) towards its equilibrium state. We shall discuss this problem in Section 5.

Let $\mathcal{F}_{s}(\mathbb{R})$, be the class of all real functions on $\mathbb{R}$ such that $g^{m}(v)$ is Hölder continuous of order $\delta$,

$$
\left\|g^{(m)}\right\|_{\delta}=\sup _{v \neq w} \frac{\left|g^{(m)}(v)-g^{(m)}(w)\right|}{|v-w|^{\delta}}<\infty
$$

the integer $m$ and the number $0<\delta \leq 1$ are such that $m+\delta=s$, and $g^{(m)}$ denotes the $m$ th derivative of $g$. In this paper, by a weak solution of the initial value problem for equation (13), corresponding to the initial measure $\mu_{0}(\mathrm{~d} v) \in \mathcal{M}_{2}$ we shall mean any measure $\mu \in C^{1}\left(\mathbb{R}_{t}^{+}, \mathcal{M}_{2}\right)$ satisfying

$$
\begin{aligned}
\frac{\mathrm{d}}{\mathrm{d} t} \int_{\mathbb{R}} \varphi(v) \mu(t, \mathrm{~d} v) & =\left(Q_{\gamma}(\mu), \varphi\right) \\
& =\frac{1}{2} \int_{\mathbb{R}^{+}} \mathrm{d} \theta \beta(\theta) \int_{\mathbb{R}^{2}}|v-w|\left[\varphi\left(v^{\prime}\right)+\varphi\left(w^{\prime}\right)-\varphi(v)-\varphi(w)\right] \mu(t, \mathrm{~d} v) \mu(t, \mathrm{~d} w)
\end{aligned}
$$


for $t>0$ and all $\varphi \in \mathcal{F}_{2}(\mathbb{R})$, and such that for all $\varphi \in \mathcal{F}_{2}(\mathbb{R})$

$$
\lim _{t \rightarrow 0} \int_{\mathbb{R}} \varphi(v) \mu(t, \mathrm{~d} v)=\int_{\mathbb{R}} \varphi(v) \mu_{0}(\mathrm{~d} v) .
$$

Then, choosing $\varphi(v)=v$ into (18) shows that the total momentum is conserved in time. For this reason, and without loss of generality, we will consider in the rest of the paper as initial values only probability measures with momentum equal to zero.

We have:

Lemma 3.1. Let $\varphi \in \mathcal{F}_{2}(\mathbb{R})$. Then, if $v^{\prime}, w^{\prime}$ are the post-collisional velocities in (2),

$$
\varphi\left(v^{\prime}\right)-\varphi(v)=\frac{1-h}{2}(w-v) \varphi^{\prime}(v)+R(\varphi)(v, w)
$$

where

$$
|R(\varphi)(v)| \leq \frac{(1-h)^{2}}{8}(v-w)^{2}\left\|\varphi^{\prime}\right\|_{1}
$$

Moreover,

$$
\left|\varphi\left(v^{\prime}\right)+\varphi\left(w^{\prime}\right)-\varphi(v)-\varphi(w)\right| \leq \frac{1-h^{2}}{4}(v-w)^{2}\left\|\varphi^{\prime}\right\|_{1} .
$$

If in addition for some $0<\delta \leq 1, \varphi \in \mathcal{F}_{2+\delta}(\mathbb{R})$

$$
\begin{aligned}
\varphi\left(v^{\prime}\right)+\varphi\left(w^{\prime}\right)-\varphi(v)-\varphi(w)= & -\frac{1-h}{2}(w-v)^{2} \int_{0}^{1} \mathrm{~d} \lambda\left[1-\lambda \frac{1-h}{2}\right] \\
& \cdot \int_{0}^{1} \mathrm{~d} \xi \varphi^{\prime \prime}\left\{\left[\xi+(1-2 \xi) \lambda \frac{1-h}{2}\right] v+\left[1-\xi+(1-2 \xi) \lambda \frac{1-h}{2}\right] w\right\} .
\end{aligned}
$$

Proof. By the collision rule (2),

$$
v^{\prime}-v=w-w^{\prime}=\frac{1-h}{2}(w-v) .
$$

Hence, if $\varphi \in C^{1}(\mathbb{R})$,

$$
\begin{aligned}
\varphi\left(v^{\prime}\right)-\varphi(v) & =\frac{1-h}{2}(w-v) \int_{0}^{1} \varphi^{\prime}\left[v+\lambda \frac{1-h}{2}(w-v)\right] \mathrm{d} \lambda \\
& =\frac{1-h}{2}(w-v)\left\{\varphi^{\prime}(v)+\int_{0}^{1}\left\{\varphi^{\prime}\left[v+\lambda \frac{1-h}{2}(w-v)\right]-\varphi^{\prime}(v)\right\} \mathrm{d} \lambda\right\} .
\end{aligned}
$$

If now $\varphi \in \mathcal{F}_{2}(\mathbb{R})$, by (17), (20) follows. On the other hand,

$$
\varphi\left(w^{\prime}\right)-\varphi(w)=\frac{1-h}{2}(v-w) \int_{0}^{1} \varphi^{\prime}\left[w-\lambda \frac{1-h}{2}(w-v)\right] \mathrm{d} \lambda .
$$

Taking the sum of (25) and (26), and using formula (25) for the difference $\varphi^{\prime}(u)-\varphi^{\prime}\left(u_{1}\right),(23)$ follows. 
Lemma 3.1 has some immediate consequences. Since

$$
1-h\left(\theta|v-w|^{\gamma}\right)^{2} \leq 2 \theta|v-w|^{\gamma}
$$

substituting (27) into (20), we conclude that, if $\gamma \geq-3,\left(Q_{\gamma}(\mu), \varphi\right)$ is bounded for all functions $\varphi \in \mathcal{F}_{2}$, and measures $\mu \in \mathcal{M}_{3+\gamma}$. Therefore we have:

Remark 3.2. Let $-3 \leq \gamma<1$. Then, $\left(Q_{\gamma}(\mu), \varphi\right)$ is defined, among others, for functions $\varphi \in \mathcal{F}_{2}$, and measures $\mu \in \mathcal{M}_{3+\gamma}$. Moreover, let $\varphi \in \mathcal{F}_{2+\alpha}(\mathbb{R})$ be convex. Then, for any measure $\mu \in \mathcal{M}_{0}$

$$
\left(Q_{\gamma}(\mu), \varphi\right) \leq 0
$$

We prove now an existence result for the Boltzmann equation (13).

Theorem 3.3. Let the probability measure $\mu_{0} \in \mathcal{M}_{p}$, where $p>3+\gamma$ if $\gamma>0$, and $p>3$ if $\gamma<0$. Then, if $-3 \leq \gamma<1$, and $\beta$ satisfies condition (12), the initial value problem for (13) has a solution $\mu(t, \mathrm{~d} v) \in$ $C^{1}\left(\mathbb{R}_{t}^{+}, \mathcal{M}_{2}\right)$ that satisfies (18) and (19). Moreover, the solution conserves momentum, while the second moment is non increasing with time,

$$
\frac{\mathrm{d}}{\mathrm{d} t} \int_{\mathbb{R}^{2}} v^{2} \mu(t, \mathrm{~d} v)=-\frac{1}{4} \int_{\mathbb{R}^{+}} \mathrm{d} \theta \beta(\theta) \int_{\mathbb{R}^{2}}\left(1-h^{2}\right)|v-w|^{3} \mu(t, \mathrm{~d} v) \mu(t, \mathrm{~d} w) .
$$

Proof. The proof is a direct consequence of Lemma 3.1 and Remark 3.2. A proof of the existence of solutions, valid for unbounded kernels, can be obtained by using methods first applied to the Boltzmann equation with intermolecular forces of infinite range [2], by now classical arguments [22]. Existence follows by approximating the unbounded kernel in the collisional operator with a sequence of smooth kernels and then passing to the limit. The main difference here is that the classical Boltzmann $H$-theorem, which prevents formation of concentration, does not hold. In the present situation, compactness follows by tightness.

\section{The quasi elastic limit of the Boltzmann EQUation}

In [17] McNamara and Young considered a simplification of their Boltzmann equation for rigid spheres, derived formally from the Boltzmann equation itself in what they called quasi elastic limit. This equation, they defined the test-particle equation, reads

$$
\frac{\partial f(v, t)}{\partial t}=q \frac{\partial}{\partial v}\left[f(v, t) \int_{\mathbb{R}}|v-w|(v-w) f(w, t) \mathrm{d} w\right]
$$

In (30), $q$ is a positive constant linked to the coefficient of restitution of their Boltzmann equation. The same equation was derived independently some year later in $[4,5]$ in a suitable scaling limit from a one-dimensional system of $N$ particles colliding inelastically. In such a model, the collision rule between two particles, which move freely between collisions, is

$$
v^{\prime}=w+\epsilon(v-w) ; \quad w^{\prime}=v-\epsilon(v-w)
$$

If one considers the limit $N \rightarrow \infty, \epsilon \rightarrow 0$ in such a way that $N \epsilon \rightarrow q$, a formal analysis shows that the spatially homogeneous evolution of the one-particle distribution satisfies (30). Equation (30) can be generalized in many ways, one of which we will consider here. Let us introduce the nonlinear friction equation

$$
\frac{\partial f(v, t)}{\partial t}=\frac{\lambda}{2} \frac{\partial}{\partial v}\left[f(v, t) \int_{\mathbb{R}}|v-w|^{1+\gamma}(v-w) f(w, t) \mathrm{d} w\right]
$$


where $-2 \leq \gamma<1$. Equation (30) is included in (32), and corresponds to the choice $\gamma=0$. In this section, we show that (32) can be deduced rigorously by (5), in a suitable quasi elastic limit. As briefly discussed in the introduction, for any microscopic collision satisfying (2) there is a loss of kinetic energy, given by (10). If we impose that the mean amount of energy transfer is finite, we need to consider only kernels which satisfy condition (12).

Condition (12) gives a mathematical meaning to the quasi elastic collision limit of the Boltzmann equation for dissipative collisions considered in [17].

We will call it quasi elastic asymptotic of the Boltzmann equation. It consists simply in letting the rate function $\beta(\theta)$ concentrate on elastic collisions, while the mean amount of energy transfer stays bounded.

The quasi elastic asymptotic considered in [4] can be easily reconsidered in our framework. Indeed, the energy lost in a single collision of (31) is

$$
v^{\prime 2}+w^{\prime 2}-\left(v^{2}+w^{2}\right)=-2 \epsilon(1-\epsilon)(v-w)^{2} .
$$

Hence, their asymptotic $N \epsilon \rightarrow \lambda$ exactly means that in the limiting procedure one maintains finite the total amount of energy loss, while the collisions are close to be elastic.

The quasi elastic asymptotic has many aspects in common with the grazing collision asymptotic of the Boltzmann equation, which is obtained when the effect of those collisions that are grazing, i.e. collisions which result in an infinitesimal angle deflection of the particles trajectories, prevails over the effect of other collisions. In this latter case, the limiting procedure consists in letting the angular cross-section of the non cut-off Boltzmann equation concentrate on grazing collisions while the mean amount of momentum transfer is maintained finite. This problem has recently studied mainly by Villani [22-24] (see also [1]). What is shown is that in this limit the collision operator is given by the action of a nonlinear Landau-Fokker-Planck operator [15].

Let us now give a precise mathematical meaning to the quasi elastic asymptotic.

Definition 4.1. Let $\left(\beta_{n}(\theta)\right)_{n>1}, \theta \in \mathbb{R}^{+}$, be a sequence of rate functions. We say that $\left(\beta_{n}\right)$ concentrate on elastic collisions if

(i) For all $\theta_{0}>0, \beta_{n}(\theta) \rightarrow 0$ as $n \rightarrow \infty$ uniformly for $\theta \geq \theta_{0}$.

(ii)

$$
\lim _{n \rightarrow \infty} \int_{\mathbb{R}^{+}} \beta_{n}(\theta) \theta \mathrm{d} \theta=\lambda<\infty .
$$

We prove:

Theorem 4.2. Let the probability measure $\mu_{0} \in \mathcal{M}_{p}$, where $p>3+2 \gamma$ if $\gamma>0$, and $p>3$ if $\gamma<0$. Let $\left(\beta_{n}(\theta)\right)_{n>1}, \theta \in \mathbb{R}^{+}$, be a sequence of rate functions concentrating to zero in the sense of Definition 4.1. Then, if $-2 \leq \gamma<1$, for all $t>0$ the solutions $\left(\mu_{n}(\mathrm{~d} v, t)\right)$ to the dissipative Boltzmann equation converge, up to extraction of a subsequence, to a probability measure $\mu(\mathrm{d} v, t)$. The measure $\mu(t, \mathrm{~d} v)$ is a weak solution of the nonlinear friction equation (32).

Proof. The proof is easily obtained applying the same strategy as in [22] for the grazing collision asymptotic of the Boltzmann equation. In some detail, let $\left(\mu^{n}\right)_{1}^{\infty}$ be the sequence of solutions to the Boltzmann equation with rate function $\beta_{n}$ and initial datum $\mu_{0}$. By (20),

$$
\begin{aligned}
\left(Q_{\gamma}^{n}(\mu), \varphi\right)= & \int_{\mathbb{R}^{+}} \beta_{n}(\theta) \mathrm{d} \theta \int_{\mathbb{R}^{2}}|v-w|\left[\varphi\left(v^{\prime}\right)-\varphi(v)\right] \mu_{n}(t, \mathrm{~d} v) \mu_{n}(t, \mathrm{~d} w) \\
= & \int_{\mathbb{R}^{+}} \beta_{n}(\theta) \mathrm{d} \theta \int_{\mathbb{R}^{2}} \frac{1-h}{2}|v-w|(w-v) \varphi^{\prime}(v) \mu_{n}(t, \mathrm{~d} v) \mu_{n}(t, \mathrm{~d} w) \\
& +\int_{\mathbb{R}^{+}} \beta_{n}(\theta) \mathrm{d} \theta \int_{\mathbb{R}^{2}}|v-w| R(\varphi) \mu_{n}(t, \mathrm{~d} v) \mu_{n}(t, \mathrm{~d} w) .
\end{aligned}
$$


If the hypotheses of the theorem are satisfied, for all $\varphi \in \mathcal{F}_{2}$ we obtain

$$
\begin{gathered}
\lim _{n \rightarrow \infty} \int_{\mathbb{R}^{+}} \beta_{n}(\theta) \frac{1-h}{2} \mathrm{~d} \theta \int_{\mathbb{R}^{2}}|v-w|(w-v) \varphi^{\prime}(v) \mu_{n}(t, \mathrm{~d} v) \mu_{n}(t, \mathrm{~d} w)= \\
-\frac{\lambda}{2} \int_{\mathbb{R}^{2}}|v-w|^{1+\gamma}(v-w) \varphi^{\prime}(v) \mu_{n}(t, \mathrm{~d} v) \mu_{n}(t, \mathrm{~d} w),
\end{gathered}
$$

while the remaining integral in (35) vanishes in the limit. Then the result follows by tightness.

Remark 4.3. The analogy between the quasi elastic asymptotic of (5) and the grazing collision asymptotic of the Boltzmann equation is evident if we consider the grazing asymptotic of the non cut-off Kac equation (7). This problem has been studied by the author in [19]. In the grazing collision limit Kac operator is well approximated by a one-dimensional linear Fokker-Planck operator. By this analogy, a sequence of rate functions satisfying the hypotheses of Definition (4.1) can be obtained simply taking some rate functions of the grazing collision kernels of the non cut-off Kac equation. For example, we can consider, for $1<p<2$, the rate functions [19]

$$
\beta_{n}(\theta)=n \frac{1}{\theta^{p}}, \quad \text { if } \quad \theta \in\left[0, \frac{1}{n^{1 /(2-p)}}\right] ; \quad \beta_{n}(\theta)=0 \quad \text { elsewhere. }
$$

\section{The COOLIng PROCEss}

As discussed in the introduction, the study of the collapse phenomenon is mainly the most important phenomenon we need to verify. When a spatially kinetic homogeneous equation is concerned, the main effect to study is referred as the cooling process. This concentration phenomenon can be studied simply by following the time evolution of moments. The decay of the second moment given in (29) shows that there is a cooling effect for any value of the parameter interaction $\gamma$. By Remark 2.2, we will assume that the rate function satisfies both conditions (9) and (12). Moreover, for the remaining of this section we suppose the rate function satisfies the additional condition

$$
\int_{\mathbb{R}^{+}} \beta(\theta) \frac{\theta}{1+\theta} \mathrm{d} \theta=c_{\gamma}<\infty
$$

Let $E(t)=\int_{\mathbb{R}} v^{2} \mu(\mathrm{d} v, t)$. We have:

Theorem 5.1. Let the probability measure $\mu_{0} \in \mathcal{M}_{p}$, where $p>3+\gamma$ if $\gamma>0$, and $p>3$ if $\gamma<0$. Then, if $-3 \leq \gamma<1$, and $\beta$ satisfies condition (38), $E(t)$ converges to zero at least as $t^{-1}$ for $\gamma \geq-1$, and at least as $t^{-2}$ for $\gamma<-1$.

Proof. If $\gamma>-1$, by Schwarz inequality we obtain

$$
\begin{aligned}
\int_{\mathbb{R}^{+}} \beta(\theta) \frac{\theta}{1+\theta} \mathrm{d} \theta \int_{\mathbb{R}^{2}}|v-w|^{2} \mu(\mathrm{d} v, t) \mu(t, \mathrm{~d} w) \\
=\int_{\mathbb{R}^{+}} \int_{\mathbb{R}^{2}}[\beta(\theta) \theta]^{1 / 2} \frac{|v-w|^{(3+\gamma) / 2}}{\left(1+\theta|v-w|^{\gamma}\right)^{1 / 2}} \\
\cdot|v-w|^{(1-\gamma) / 2}\left(1+\theta|v-w|^{\gamma}\right)^{1 / 2} \frac{[\beta(\theta) \theta]^{1 / 2}}{1+\theta} \mu(t, \mathrm{~d} v) \mu(t, \mathrm{~d} w) \\
\leq \\
{\left[\int_{\mathbb{R}^{+}} \beta(\theta) \theta \mathrm{d} \theta \int_{\mathbb{R}^{2}} \frac{|v-w|^{(3+\gamma)}}{1+\theta|v-w|^{\gamma}} \mu(t, \mathrm{~d} v) \mu(t, \mathrm{~d} w)\right]^{1 / 2} } \\
\cdot\left[\int_{\mathbb{R}^{+}} \beta(\theta) \frac{\theta}{1+\theta} \mathrm{d} \theta \int_{\mathbb{R}^{2}}|v-w|^{1-\gamma} \frac{1+\theta|v-w|^{\gamma}}{1+\theta} \mu(t, \mathrm{~d} v) \mu(t, \mathrm{~d} w)\right]^{1 / 2} .
\end{aligned}
$$


Now we have

$$
\begin{aligned}
\int_{\mathbb{R}^{2}}|v-w|^{1-\gamma} \frac{1+\theta|v-w|^{\gamma}}{1+\theta} \mu(t, \mathrm{~d} v) \mu(t, \mathrm{~d} w) & \leq \int_{|v-w| \leq 1} \mu(t, \mathrm{~d} v) \mu(t, \mathrm{~d} w)+\int_{|v-w|>1}|v-w| \mu(t, \mathrm{~d} v) \mu(t, \mathrm{~d} w) \\
& \leq 1+\left[\int_{\mathbb{R}^{2}}|v-w|^{2} \mu(t, \mathrm{~d} v) \mu(t, \mathrm{~d} w)\right]^{1 / 2}
\end{aligned}
$$

Considering that $E(t)$ is non increasing, we get

$$
\int_{\mathbb{R}^{2}}|v-w|^{2} \mu(t, \mathrm{~d} v) \mu(t, \mathrm{~d} w)=2 E(t) \leq 2 E(0) .
$$

Finally

$$
\begin{aligned}
2 c_{\gamma} E(t) & =\int_{\mathbb{R}^{+}} \beta(\theta) \frac{\theta}{1+\theta} \mathrm{d} \theta \int_{\mathbb{R}^{2}}|v-w|^{2} \mu(\mathrm{d} v, t) \mu(t, \mathrm{~d} w) \\
& \leq\left[\int_{\mathbb{R}^{+}} \beta(\theta) \theta \mathrm{d} \theta \int_{\mathbb{R}^{2}} \frac{|v-w|^{(3+\gamma)}}{1+\theta|v-w|^{\gamma}} \mu(t, \mathrm{~d} v) \mu(t, \mathrm{~d} w)\right]^{1 / 2}\left[1+(2 E(0))^{1 / 2}\right]^{1 / 2}
\end{aligned}
$$

Applying inequality (42) into (29), we get

$$
\begin{aligned}
\frac{\mathrm{d} E}{\mathrm{~d} t} & =-\frac{1}{4} \int_{\mathbb{R}^{+}} \beta(\theta) \theta \mathrm{d} \theta \int_{\mathbb{R}^{2}}(1+h) \frac{|v-w|^{(3+\gamma)}}{1+\theta|v-w|^{\gamma}} \mu(t, \mathrm{~d} v) \mu(t, \mathrm{~d} w) \\
& \leq-\frac{\lambda c_{\gamma}^{2}}{1+(2 E(0))^{1 / 2}} E(t)^{2}
\end{aligned}
$$

which implies that $E(t)$ converges to zero at least as $t^{-1}$. The constant $\lambda$ in $(43)$ is defined by

$$
\int_{\mathbb{R}^{+}} \beta(\theta) \theta \mathrm{d} \theta=\lambda
$$

Let now $\gamma<-1$, and let $\sigma=-\gamma$. By Hölder inequality we get

$$
\begin{aligned}
\int_{\mathbb{R}^{2}}|v-w|^{2} \mu(\mathrm{d} v, t) \mu(t, \mathrm{~d} w) & =\int_{\mathbb{R}^{2}} \frac{|v-w|^{2}}{\left(\theta+|v-w|^{\sigma}\right)^{2 / 3}}\left(\theta+|v-w|^{\sigma}\right)^{2 / 3} \mu(t, \mathrm{~d} v) \mu(t, \mathrm{~d} w) \\
& \leq\left[\int_{\mathbb{R}^{2}} \frac{|v-w|^{3}}{\theta+|v-w|^{\sigma}} \mu(t, \mathrm{~d} v) \mu(t, \mathrm{~d} w)\right]^{2 / 3}\left[\int_{\mathbb{R}^{2}}\left(\theta+|v-w|^{\sigma}\right)^{2} \mu(t, \mathrm{~d} v) \mu(t, \mathrm{~d} w)\right]^{1 / 3}
\end{aligned}
$$

Since $\sigma \geq 1, \int_{\mathbb{R}}|v|^{2 \sigma} \mu(t, \mathrm{~d} v)$ is non increasing. Hence

$$
\int_{\mathbb{R}^{2}}\left(\theta+|v-w|^{\sigma}\right)^{2} \mu(t, \mathrm{~d} v) \mu(t, \mathrm{~d} w) \leq 2 \theta^{2}+2 c_{\sigma} \int_{\mathbb{R}}|v|^{2 \sigma} \mu_{0}(\mathrm{~d} v),
$$

where $c_{\sigma}$ is a suitable positive constant. Let us substitute (46) into (45). We obtain

$$
\int_{\mathbb{R}^{2}} \frac{|v-w|^{3}}{\theta+|v-w|^{\sigma}} \mu(t, \mathrm{~d} v) \mu(t, \mathrm{~d} w) \geq \frac{[2 E(t)]^{3 / 2}}{\theta+c_{\sigma} \int_{\mathbb{R}}|v|^{2 \sigma} \mu_{0}(\mathrm{~d} v)} .
$$


Applying inequality (47) into (29), we get

$$
\begin{aligned}
\frac{\mathrm{d} E}{\mathrm{~d} t} & \leq-\frac{1}{4} \int_{\mathbb{R}^{+}} \beta(\theta) \theta \mathrm{d} \theta \int_{\mathbb{R}^{2}}(1+h) \frac{|v-w|^{3}}{\theta+|v-w|^{\sigma}} \mu(t, \mathrm{~d} v) \mu(t, \mathrm{~d} w) \\
& \leq-\frac{1}{4} \int_{\mathbb{R}^{+}} \beta(\theta) \frac{\theta}{\theta+c_{\sigma} \int_{\mathbb{R}}|v|^{2 \sigma} \mu_{0}(\mathrm{~d} v)} \mathrm{d} \theta[2 E(t)]^{3 / 2}
\end{aligned}
$$

This implies that $E(t)$ converges to zero at least as $t^{-2}$.

Let us investigate in more details the decay of $E(t)$ for $\gamma \geq-1$. It is immediate to conclude that in this case there is no cooling in finite time. In fact, let us consider the situation in which the initial measure has bounded support, say $\int_{|v| \geq R} \mu_{0}(\mathrm{~d} v)=0$. Then, since the support is non increasing with time,

$$
\begin{aligned}
\int_{\mathbb{R}^{+}} \beta(\theta) \mathrm{d} \theta \int_{\mathbb{R}^{2}}|v-w|^{3}\left(1-h^{2}\right) \mu(t, \mathrm{~d} v) \mu(t, \mathrm{~d} w) & \leq(2 R)^{1+\gamma} \int_{\mathbb{R}^{+}} \beta(\theta) \theta \mathrm{d} \theta \int_{\mathbb{R}^{2}}|v-w|^{2} \mu(t, \mathrm{~d} v) \mu(t, \mathrm{~d} w) \\
& =(2 R)^{1+\gamma} \lambda 2 E(t) .
\end{aligned}
$$

Hence, $E(t)$ satisfies the inequality

$$
\frac{\mathrm{d} E}{\mathrm{~d} t} \geq-\frac{1}{2}(2 R)^{1+\gamma} \lambda E(t)
$$

and this implies that the convergence to zero can not be more than exponential with time. Hence we proved the following

Theorem 5.2. Let $\gamma \geq-1$. Then, if the probability measure $\mu_{0}$ is compactly supported, the convergence to zero of the second moment $E(t)$ is at most exponential in time.

Remark 5.3. The result of Theorem 5.1 can easily be extended to nonlinear friction equations. In this case, for $\gamma \geq-1$ the precise decay to zero of $E(t)$ can be recovered by estimates similar to that used in [4]. In addition, by using the technique developed in [4], when $\gamma \geq-1$, Theorem 5.2 can be extended to initial data of unbounded support. From a physical point of view, the importance of the result is linked to the fact that for a wide range of values of $\gamma$ in the expression of the coefficient of restitution, including anomalous granular materials, there is no finite time cooling even if the support of the velocities is initially a small interval like $[-\epsilon, \epsilon]$. Finally, the result of Theorem 5.2 remains valid for any value of the parameter $\gamma$, provided $\beta(\theta)$ is integrable.

The previous analysis can not be pushed easily further, and the possibility of finite time cooling for $\gamma<-1$ remains an open problem. In the next section finite time cooling will be investigated for the nonlinear friction equations (32), by means of a detailed study of their similarity solutions.

\section{Similarity SOlUtions of NONLINEAR FRICTION EQUATIONS}

To understand the grouping of particles, both [17] and [4] considered self-similar solutions to the test-particle equation (30). Here we face the same problem for general nonlinear friction equations by means of the classical methods developed in [3].

Let $f(v, t)$ be a solution to (32). Then, for $\alpha=\alpha(t) \geq 0$ and $\tau=\tau(t) \geq 0$, let $g$ be defined through the relation

$$
f(v, t)=\frac{1}{\alpha} g\left(\frac{v}{\alpha}, \tau\right)=\frac{1}{\alpha} g\left(v^{\prime}, \tau\right)
$$


Then, since

$$
\frac{\partial}{\partial t} f(v, t)=\frac{\tau^{\prime}}{\alpha} \frac{\partial}{\partial \tau} g\left(v^{\prime}, \tau\right)-\frac{\alpha^{\prime}}{\alpha^{2}} \frac{\partial}{\partial v^{\prime}}\left(v^{\prime} g\left(v^{\prime}, \tau\right)\right)
$$

by the position

$$
\tau(t)=-\log \alpha(t)
$$

we obtain

$$
\begin{aligned}
\frac{\partial}{\partial t} f(v, t) & =-\frac{\alpha^{\prime}}{\alpha^{2}}\left[\frac{\partial}{\partial \tau} g\left(v^{\prime}, \tau\right)+\frac{\partial}{\partial v^{\prime}}\left(v^{\prime} g\left(v^{\prime}, \tau\right)\right)\right] \\
& =\alpha^{\gamma} \frac{\lambda}{2} \frac{\partial}{\partial v^{\prime}}\left[g\left(v^{\prime}, \tau\right) \int_{\mathbb{R}}\left|v^{\prime}-w^{\prime}\right|^{1+\gamma}\left(v^{\prime}-w^{\prime}\right) g\left(w^{\prime}, \tau\right) \mathrm{d} w^{\prime}\right] .
\end{aligned}
$$

Finally, for all $-2 \leq \gamma<1, \gamma \neq-1$, setting

$$
\alpha(t)=\left(\frac{1}{1+(1+\gamma) t}\right)^{1 /(1+\gamma)}
$$

which implies

$$
\alpha^{\gamma}=-\frac{\alpha^{\prime}}{\alpha^{2}}
$$

we obtain at once that $g\left(v^{\prime}, \tau\right)$ satisfies

$$
\frac{\partial}{\partial \tau} g\left(v^{\prime}, \tau\right)=\frac{\partial}{\partial v^{\prime}}\left[g\left(v^{\prime}, \tau\right) \frac{\lambda}{2} \int_{\mathbb{R}}\left|v^{\prime}-w^{\prime}\right|^{1+\gamma}\left(v^{\prime}-w^{\prime}\right) g\left(w^{\prime}, \tau\right) \mathrm{d} w^{\prime}-v^{\prime} g\left(v^{\prime}, \tau\right)\right],
$$

with $\alpha(t) \geq 0$ decreasing, $\tau(t)$ increasing from 0 to $\infty$, with $\tau(0)=0$. We remark here the different behavior of $\alpha(t)$, for different values of $\gamma$. If $\gamma>-1, \alpha(t)$ decreases from 1 to 0 , and tends to zero as time goes to $\infty$ at a rate $t^{1 /(1+\gamma)}$. If $\gamma<-1, \alpha(t)$ decreases from 1 to 0 , and becomes equal to zero in finite time, $\alpha(t)=0$ for $t \geq 1 /(1+|\gamma|)$.

Let us look for stationary solutions to equation (57). The existence of stationary solutions different from concentration are made possible by the structure of the right-hand side of (57). This right-hand side represents a balance between a nonlinear friction and an anti drift that are responsible of opposite effects. Stationary solutions to (57) solve

$$
g(v)\left[\frac{\lambda}{2} \int_{\mathbb{R}}|v-w|^{1+\gamma}(v-w) g(w) \mathrm{d} w-v\right]=0 .
$$

Following the idea of [17] and [4], we look for solutions which are sums of Dirac masses. The simplest of these solution with zero momentum, is clearly given by two masses located symmetrically with respect to the origin

$$
g_{\infty}(v)=c \delta\left(v+v_{0}\right)+c \delta\left(v-v_{0}\right), \quad c>0, v_{0}>0 .
$$

For any smooth function $\varphi$ supported in $(-a, a)$, with $a>v_{0}$, and for any $\gamma,-2<\gamma<1, g \neq-1$, we verify that $g_{\infty}$ is a solution of

$$
\int v g(v) \varphi(v) \mathrm{d} v=\frac{\lambda}{2} \int g(v)\left[\int_{-a}^{v}|v-w|^{2+\gamma} g(w) \mathrm{d} w-\int_{v}^{a}|v-w|^{2+\gamma} g(w) \mathrm{d} w\right] \varphi(v) \mathrm{d} v .
$$


It is clear that

$$
\int v g_{\infty}(v) \varphi(v) \mathrm{d} v=-c v_{0} \varphi\left(-v_{0}\right)+c v_{0} \varphi\left(v_{0}\right)
$$

Let us set

$$
G(v)=\int_{-a}^{v}|v-w|^{2+\gamma} g_{\infty}(w) \mathrm{d} w-\int_{v}^{a}|v-w|^{2+\gamma} g_{\infty}(w) \mathrm{d} w
$$

One obtains

$$
G(v)= \begin{cases}-c\left|v+v_{0}\right|^{2+\gamma}-c\left|v-v_{0}\right|^{2+\gamma} & \text { if } \quad v \leq-v_{0} \\ c\left|v+v_{0}\right|^{2+\gamma}-c\left|v-v_{0}\right|^{2+\gamma} & \text { if } \quad-v_{0} \leq v \leq v_{0} \\ c\left|v+v_{0}\right|^{2+\gamma}+c\left|v-v_{0}\right|^{2+\gamma} & \text { if } \quad v \geq v_{0}\end{cases}
$$

Whenever $-2<\gamma<-1$, or $-1<\gamma<2, G(v)$ is continuous in $-v_{0}$ and $v_{0}$, and

$$
G\left(-v_{0}\right)=-c\left(2 v_{0}\right)^{2+\gamma} ; \quad G\left(v_{0}\right)=c\left(2 v_{0}\right)^{2+\gamma} .
$$

Finally,

$$
\int G(v) g_{\infty}(v) \varphi(v) \mathrm{d} v=-c^{2} \frac{\lambda}{2}\left(2 v_{0}\right)^{2+\gamma} \varphi\left(-v_{0}\right)+c^{2} \frac{\lambda}{2}\left(2 v_{0}\right)^{2+\gamma} \varphi\left(v_{0}\right)
$$

Let us choose $c$ and $v_{0}$ such that

$$
v_{0}=c \frac{\lambda}{2}\left(2 v_{0}\right)^{2+\gamma}
$$

Then (61) and (65) coincide, and $g_{\infty}$ is a solution to (60). The values of $c$ and $v_{0}$ are easily determined considering that $2 c$ is the total mass. If we impose that $g_{\infty}$ is a probability density, $c=1 / 2$. Consequently,

$$
v_{0}=\left(\frac{1}{\lambda 2^{\gamma}}\right)^{1 /(1+\gamma)} .
$$

So we proved:

Theorem 6.1. Let $-2<\gamma<1, \gamma \neq-1$, and let $g_{\infty}(v)$ be the probability density

$$
g_{\infty}(v)=-\frac{1}{2} \delta\left(v+\left(\frac{1}{\lambda 2^{\gamma}}\right)^{1 /(1+\gamma)}\right)+\frac{1}{2} \delta\left(v-\left(\frac{1}{\lambda 2^{\gamma}}\right)^{1 /(1+\gamma)}\right)
$$

Then, $g_{\infty}(v)$ is a stationary solution of equation (58).

Theorem 6.1 has interesting consequences. It is immediate to conclude that any stationary solution to (58), reverting to the old variables, is a similarity solution to (32). This solution, given by

$$
f_{s}(v, t)=\frac{1}{\alpha(t)} g_{\infty}\left(\frac{v}{\alpha(t)}\right)
$$

represents a homogeneous cooling state of the nonlinear friction equation. Looking at the form of the function $\alpha(t)$, we conclude that, while for $\gamma>-1$ there is no cooling in finite time, when $\gamma<-1$ we have cooling in 
finite time, and the solution concentrates at time $t_{c}=1 / 1+|\gamma|$. This fact is in favor of the conjecture that any solution to the nonlinear friction equation corresponding to $\gamma<-1$ ceases to exist after a finite time, but a general proof is still missing.

We end this section by showing that the case $\gamma=-2$ which was excluded from the previous analysis, can be treated in a direct way. As usual, let us suppose that the stationary state is a probability density, and let us define, for $a>0$,

$$
g_{\infty}(v)=\frac{1}{2 a} I(-a, a),
$$

where $I(\Omega)$ is the characteristic function of the set $\Omega$. Then

$$
\int v g_{\infty}(v) \varphi(v) \mathrm{d} v=\frac{1}{2 a} \int_{-a}^{a} v \varphi(v) \mathrm{d} v
$$

while

$$
G(v)=\int_{-a}^{v} g_{\infty}(w) \mathrm{d} w-\int_{v}^{a} g_{\infty}(w) \mathrm{d} w=\frac{v}{a}
$$

Therefore one obtains

$$
\frac{\lambda}{2} \int G(v) g_{\infty}(v) \varphi(v) \mathrm{d} v=\frac{\lambda}{4 a^{2}} \int_{-a}^{a} v \varphi(v) \mathrm{d} v
$$

which implies that (61) and (65) coincide, provided $\lambda=2 a$. Thus

$$
g_{\infty}(v)=\frac{1}{\lambda} I\left(-\frac{1}{2 \lambda}, \frac{1}{2 \lambda}\right),
$$

is a solution to (60), with $\gamma=-2$. Reverting to the old variables, we obtain the homogeneous cooling state of the nonlinear friction equation with $\gamma=-2$. We remark here the difference between the case $\gamma>-2$, where the homogeneous cooling is represented by two masses located symmetrically with respect to the origin, and moving towards the origin itself, and the case $\gamma=-2$, where the homogeneous cooling is a continuous uniform distribution symmetric with respect to the origin, concentrating in finite time.

Acknowledgements. This paper was concluded while the author was visiting the École Normale Supérieure of Paris. He would like to express his sincere gratitude to C. Villani and B. Perthame for their kind hospitality and for several discussions on dissipative equations. Financial supports both from the TMR project "Asymptotic Methods in Kinetic Theory", No. ERBFRMXCT 970157, funded by the EC., and from the Italian MURST, project "Mathematical Problems in Kinetic Theories" are gratefully acknowledged.

\section{REFERENCES}

[1] R. Alexandre and C. Villani, On the Boltzmann equation for long range interactions and the Landau approximation in plasma physics. Preprint DMA, École Normale Supérieure (1999).

[2] L. Arkeryd, Intermolecular forces of infinite range and the Boltzmann equation. Arch. Rational Mech. Anal. 77 (1981) $11-21$.

[3] G.I. Barenblatt, Scaling, Self-similarity, and Intermediate Asymptotics. Cambridge Univ. Press, New York (1996).

[4] D. Benedetto, E. Caglioti and M. Pulvirenti, A kinetic equation for granular media. RAIRO Modél. Math. Anal. Numér. 31 (1997) 615-641.

[5] D. Benedetto, E. Caglioti and M. Pulvirenti, Erratum: A kinetic equation for granular media [RAIRO Modél. Math. Anal. Numér. 31 (1997) 615-641]. ESAIM: M2AN 33 (1999) 439-441.

[6] C. Bizon, J.B. Shattuck, M.D. Swift, W.D. McCormick and H.L. Swinney, Pattern in 2D vertically oscillated granular layers: simulation and experiments. Phys. Rev. Lett. 80 (1998) 57-60. 
[7] A.V. Bobylev, J.A-Carrillo and I. Gamba, On some properties of kinetic and hydrodynamics equations for inelastic interactions. J. Statist. Phys. 98 (2000) 743-773.

[8] C. Cercignani, R. Illner and M. Pulvirenti, The mathematical theory of dilute gases. Springer Ser. Appl. Math. Sci. 106, Springer-Verlag, New York (1994).

[9] L. Desvillettes, About the regularizing properties of the non-cut-off Kac equation. Comm. Math. Phys. 168 (1995) $417-440$.

[10] Y. Du, H. Li and L.P. Kadanoff, Breakdown of hydrodynamics in a one-dimensional system of inelastic particles. Phys. Rev. Lett. 74 (1995) 1268-1271.

[11] D. Goldman, M.D. Shattuck, C. Bizon, W.D. McCormick, J.B. Swift and H.L. Swinney, Absence of inelastic collapse in a realistic three ball model. Phys. Rev. E 57 (1998) 4831-4833.

[12] I. Goldhirsch, Scales and kinetics of granular flows. Chaos 9 (1999) 659-672.

[13] M. Kac, Probability and related topics in the physical sciences. New York (1959).

[14] L. Kantorovich, On translation of mass (in Russian). Dokl. AN SSSR 37 (1942) 227-229.

[15] L. Landau, Die kinetische Gleichung für den Fall Coulombscher Wechselwirkung. Phys. Z. Sowjet. 10 (1936) 154. Trad.: The transport equation in the case of Coulomb interactions, in Collected papers of L.D. Landau, D. ter Haar Ed., Pergamon Press, Oxford (1981) 163-170.

[16] S. McNamara and W.R. Young, Inelastic collapse and clumping in a one-dimensional granular medium. Phys. Fluids A 4 (1992) 496-504.

[17] S. McNamara and W.R. Young, Kinetics of a one-dimensional granular medium in the quasi-elastic limit. Phys. Fluids A 5 (1993) 34-45.

[18] G. Naldi, L. Pareschi and G. Toscani, Spectral methods for a singular Boltzmann equation for granular flows and numerical quasi elastic limit. Preprint (2000).

[19] G. Toscani, The grazing collision asymptotic of the non cut-off Kac equation. RAIRO Modél. Math. Anal. Numér. 32 (1998) $763-772$.

[20] I. Vaida, Theory of statistical Inference and Information. Kluwer Academic Publishers, Dordrecht (1989).

[21] L.N. Vasershtein, Markov processes on countable product space describing large systems of automata (in Russian). Problemy Peredachi Informatsii 5 (1969) 64-73.

[22] C. Villani, Contribution à l'étude mathématique des équations de Boltzmann et de Landau en théorie cinétique des gaz et des plasmas. Ph.D. thesis, Univ. Paris-Dauphine (1998).

[23] C. Villani, On a new class of weak solutions to the spatially homogeneous Boltzmann and Landau equations. Arch. Rational Mech. Anal. 143 (1998) 273-307.

[24] C. Villani, Contribution à l'étude mathématique des collisions en théorie cinétique. Ceremade, Paris IX-Dauphine, January 24 (2000).

[25] V.M. Zolotarev, Probability Metrics. Theory Probab. Appl. 28 (1983) 278-302.

To access this journal online:

www.edpsciences.org 